\title{
An Existence Theorem for Fractional Hybrid Differential Inclusions of Hadamard Type with Dirichlet Boundary Conditions
}

\author{
Bashir Ahmad ${ }^{1}$ and Sotiris K. Ntouyas ${ }^{2}$ \\ ${ }^{1}$ Department of Mathematics, Faculty of Science, King Abdulaziz University, P.O. Box 80203, Jeddah 21589, Saudi Arabia \\ ${ }^{2}$ Department of Mathematics, University of Ioannina, 45110 Ioannina, Greece
}

Correspondence should be addressed to Bashir Ahmad; bashirahmad_qau@yahoo.com

Received 5 May 2014; Accepted 5 June 2014; Published 19 June 2014

Academic Editor: Guotao Wang

Copyright (C) 2014 B. Ahmad and S. K. Ntouyas. This is an open access article distributed under the Creative Commons Attribution License, which permits unrestricted use, distribution, and reproduction in any medium, provided the original work is properly cited.

This paper studies the existence of solutions for a boundary value problem of nonlinear fractional hybrid differential inclusions by using a fixed point theorem due to Dhage (2006). The main result is illustrated with the aid of an example.

\section{Introduction}

The intensive development of fractional calculus in recent years clearly indicates the popularity of the subject. It has been mainly due to applications of the subject in various fields such as physics, mechanics, chemistry, and engineering [1-3]. In particular, the tools of fractional calculus have considerably improved the modelling techniques and several important models describing biological, ecological, and engineering phenomena are now based on fractional derivatives and integrals. Another factor attracting the attention of many scientists is the nonlocal nature of fractional-order operators which accounts for the hereditary properties of many materials and processes.

Much of the work on fractional differential equations involves either Riemann-Liouville derivative or Caputo derivative; for instance, see [4-33] and the references therein. However, there is another concept of fractional derivative in the literature which was introduced by Hadamard in 1892 [34]. This derivative is known as Hadamard fractional derivative and differs from aforementioned derivatives in the sense that the kernel of the integral in its definition contains logarithmic function of arbitrary exponent. Further details of Hadamard fractional derivatives and integrals can be found in [2].
In this paper, we study a Dirichlet boundary value problem of nonlinear fractional hybrid differential inclusions given by

$$
\begin{gathered}
D^{\alpha}\left(\frac{x(t)}{f(t, x(t))}\right) \in F(t, x(t)), \quad 1<t<e, \quad 1<\alpha \leq 2, \\
x(1)=x(e)=0
\end{gathered}
$$

where $D^{\alpha}$ is the Hadamard fractional derivative, $f \in$ $C([1, e] \times \mathbb{R}, \mathbb{R} \backslash\{0\}), F:[1, e] \times \mathbb{R} \rightarrow \mathscr{P}(\mathbb{R})$ is a multivalued map, and $\mathscr{P}(\mathbb{R})$ is the family of all nonempty subsets of $\mathbb{R}$.

The main objective of the present study is to establish an existence result for the problem (1) under Lipschitz and Carathéodory conditions by applying a fixed point theorem in Banach algebras due to Dhage [35]. Some recent details on hybrid fractional differential equations can be found in [36$40]$ and the references cited therein. We emphasize that our work is new in the present configuration and contributes to the present literature on Hadamard type fractional differential equations and inclusions [41-44].

The paper is organized as follows: in Section 2 we recall some preliminary facts that we need in the sequel and Section 3 contains our main result. 


\section{Preliminaries}

\subsection{Fractional Calculus}

Definition 1 (see [2]). The Hadamard derivative of fractional order $q$ for a function $g:[1, \infty) \rightarrow \mathbb{R}$ is defined as

$$
\begin{array}{r}
D^{q} g(t)=\frac{1}{\Gamma(n-q)}\left(t \frac{d}{d t}\right)^{n} \int_{1}^{t}\left(\log \frac{t}{s}\right)^{n-q-1} \frac{g(s)}{s} d s, \\
n-1<q<n, \quad n=[q]+1,
\end{array}
$$

where $[q]$ denotes the integer part of the real number $q$ and $\log (\cdot)=\log _{e}(\cdot)$.

Definition 2 (see [2]). The Hadamard fractional integral of order $q$ for a function $g$ is defined as

$$
I^{q} g(t)=\frac{1}{\Gamma(q)} \int_{1}^{t}\left(\log \frac{t}{s}\right)^{q-1} \frac{g(s)}{s} d s, \quad q>0,
$$

provided the integral exists.

Lemma 3. Let $y \in C([1, e], \mathbb{R})$. Then the integral solution of the problem

$$
\begin{gathered}
D^{\alpha}\left(\frac{x(t)}{f(t, x(t))}\right)=y(t), \quad 1<t<e, 1<\alpha \leq 2, \\
x(1)=x(e)=0,
\end{gathered}
$$

is given by

$x(t)$

$$
\begin{aligned}
=f(t, x(t))( & \frac{1}{\Gamma(\alpha)} \int_{1}^{t}\left(\log \frac{t}{s}\right)^{\alpha-1} \frac{y(s)}{s} d s \\
& \left.-(\log t)^{\alpha-1} \frac{1}{\Gamma(\alpha)} \int_{1}^{e}\left(\log \frac{e}{s}\right)^{\alpha-1} \frac{y(s)}{s} d s\right), \\
& t \in[1, e] .
\end{aligned}
$$

Proof. As argued in [2], the solution of Hadamard differential equation in (4) can be written as

$$
\begin{aligned}
x(t)=f(t, x(t))( & \frac{1}{\Gamma(\alpha)} \int_{1}^{t}\left(\log \frac{t}{s}\right)^{\alpha-1} \frac{y(s)}{s} d s \\
& \left.+c_{1}(\log t)^{\alpha-1}+c_{2}(\log t)^{\alpha-2}\right),
\end{aligned}
$$

where $c_{1}, c_{2} \in \mathbb{R}$ are arbitrary constants. Using the given boundary conditions in (6), we find that

$$
c_{2}=0, \quad c_{1}=-\frac{1}{\Gamma(\alpha)} \int_{1}^{e}\left(\log \frac{e}{s}\right)^{\alpha-1} \frac{y(s)}{s} d s .
$$

Substituting the values of $c_{1}, c_{2}$ in (6), we obtain (5).
Remark 4. It is interesting to note that solution (5) for $\alpha=$ 2 corresponds to the one for a Dirichlet boundary value problem of Cauchy-Euler type hybrid differential equation:

$$
t^{2} \frac{d^{2}}{d t^{2}}\left(\frac{x(t)}{f(t, x(t))}\right)+t \frac{d}{d t}\left(\frac{x(t)}{f(t, x(t))}\right)=y(t)
$$

2.2. Multivalued Analysis. Let us recall some basic definitions on multivalued maps $[45,46]$.

For a normed space $(X,\|\cdot\|)$, let $\mathscr{P}_{\mathrm{cl}}(X)=\{Y \in \mathscr{P}(X)$ : $Y$ is closed $\}, \mathscr{P}_{b}(X)=\{Y \in \mathscr{P}(X): Y$ is bounded $\}$, $\mathscr{P}_{\mathrm{cp}}(X)=\{Y \in \mathscr{P}(X): Y$ is compact $\}$, and $\mathscr{P}_{\mathrm{cp}, \mathrm{cv}}(X)=$ $\{Y \in \mathscr{P}(X): Y$ is compact and convex $\}$. A multivalued map $G: X \rightarrow \mathscr{P}(X)$ is convex (closed) valued if $G(x)$ is convex (closed) for all $x \in X$. The map $G$ is bounded on bounded sets if $G(\mathbb{B})=\cup_{x \in \mathbb{B}} G(x)$ is bounded in $X$ for all $\mathbb{B} \in \mathscr{P}_{b}(X)$ (i.e., $\sup _{x \in \mathbb{B}}\{\sup \{|y|: y \in G(x)\}\}<\infty$ ). $G$ is called upper semicontinuous (u.s.c.) on $X$ if for each $x_{0} \in X$, the set $G\left(x_{0}\right)$ is a nonempty closed subset of $X$, and if for each open set $N$ of $X$ containing $G\left(x_{0}\right)$, there exists an open neighborhood $\mathcal{N}_{0}$ of $x_{0}$ such that $G\left(\mathcal{N}_{0}\right) \subseteq N$. $G$ is said to be completely continuous if $G(\mathbb{B})$ is relatively compact for every $\mathbb{B} \in \mathscr{P}_{b}(X)$. If the multivalued map $G$ is completely continuous with nonempty compact values, then $G$ is u.s.c. if and only if $G$ has a closed graph; that is, $x_{n} \rightarrow x_{*}$, $y_{n} \rightarrow y_{*}$, and $y_{n} \in G\left(x_{n}\right)$ imply $y_{*} \in G\left(x_{*}\right)$. $G$ has a fixed point if there is $x \in X$ such that $x \in G(x)$. The fixed point set of the multivalued operator $G$ will be denoted by Fix $G$. A multivalued map $G:[0 ; 1] \rightarrow \mathscr{P}_{\mathrm{cl}}(\mathbb{R})$ is said to be measurable if for every $y \in \mathbb{R}$, the function

$$
t \longmapsto d(y, G(t))=\inf \{|y-z|: z \in G(t)\}
$$

is measurable.

Let $C([1, e], \mathbb{R})$ denote a Banach space of continuous functions from $[1, e]$ into $\mathbb{R}$ with the norm $\|x\|=$ $\sup _{t \in[1, e]}|x(t)|$. Let $L^{1}([1, e], \mathbb{R})$ be the Banach space of measurable functions $x:[1, e] \rightarrow \mathbb{R}$ which are Lebesgue integrable and normed by $\|x\|_{L^{1}}=\int_{1}^{e}|x(t)| d t$.

Definition 5. A multivalued map $F:[1, e] \times \mathbb{R} \rightarrow \mathscr{P}(\mathbb{R})$ is said to be Carathéodory if

(i) $t \mapsto F(t, x)$ is measurable for each $x \in \mathbb{R}$;

(ii) $x \mapsto F(t, x)$ is upper semicontinuous for almost all $t \in[1, e]$.

Further, a Carathéodory function $F$ is called $L^{1}$ Carathéodory if

(iii) there exists a function $g \in L^{1}\left([1, e], \mathbb{R}^{+}\right)$such that

$$
\|F(t, x)\|=\sup \{|v|: v \in F(t, x)\} \leq g(t),
$$

for all $x \in \mathbb{R}$ and for a.e. $t \in[1, e]$. by

For each $y \in C([1, e], \mathbb{R})$, define the set of selections of $F$

$$
\begin{array}{r}
S_{F, y}:=\left\{v \in L^{1}([1, e], \mathbb{R}): v(t) \in F(t, y(t))\right. \\
\text { for a.e. } t \in[1, e]\} .
\end{array}
$$


The following lemma is used in the sequel.

Lemma 6 (see [47]). Let $X$ be a Banach space. Let $F:[1, e] \times$ $\mathbb{R} \rightarrow \mathscr{P}_{c p, c v}(X)$ be an $L^{1}$-Carathéodory multivalued map, and let $\Theta$ be a linear continuous mapping from $L^{1}([1, e], X)$ to $C([1, e], X)$. Then the operator

$$
\begin{gathered}
\Theta \circ S_{F}: C([1, e], X) \longrightarrow \mathscr{P}_{c p, c v}(C([1, e], X)), \\
x \longmapsto\left(\Theta \circ S_{F}\right)(x)=\Theta\left(S_{F, x}\right)
\end{gathered}
$$

is a closed graph operator in $C([1, e], X) \times C([1, e], X)$.

The following fixed point theorem due to Dhage [35] is fundamental in the proof of our main result.

Lemma 7. Let $X$ be a Banach algebra, let $A: X \rightarrow X$ be a single-valued, and let $B: X \rightarrow \mathscr{P}_{c p, c v}(X)$ be a multivalued operator satisfying the following:

(a) A is single-valued Lipschitz with a Lipschitz constant $k$,

(b) $B$ is compact and upper semicontinuous,

(c) $2 M k<1$, where $M=\|B(X)\|$.

Then either

(i) the operator inclusion $x \in A x B x$ has a solution, or

(ii) the set $\mathscr{E}=\{u \in X \mid \mu u \in A u B u, \mu>1\}$ is unbounded.

\section{Main result}

Definition 8. A function $x \in A C^{1}([1, e], \mathbb{R})$ is called a solution of the problem (1) if there exists a function $v \in$ $L^{1}([1, e], \mathbb{R})$ with $v(t) \in F(t, x(t))$, a.e. on $[1, e]$ such that $D^{\alpha}(x(t) / f(t, x(t)))=v(t)$, a.e. on $[1, e]$ and $x(1)=x(e)=0$.

Theorem 9. Assume that

$\left(\mathrm{H}_{1}\right)$ the function $f:[1, e] \times \mathbb{R} \rightarrow \mathbb{R} \backslash\{0\}$ is continuous and there exists a bounded function $\phi$, with bound $\|\phi\|$, such that $\phi(t)>0$, a.e $t \in[1, e]$ and

$$
\begin{gathered}
|f(t, x)-f(t, y)| \leq \phi(t)|x(t)-y(t)| \\
\text { a.e. } t \in[1, e], \forall x, y \in \mathbb{R} ;
\end{gathered}
$$

$\left(\mathrm{H}_{2}\right) F:[1, e] \times \mathbb{R} \rightarrow \mathscr{P}(\mathbb{R})$ is $L^{1}$-Carathéodory and has nonempty compact and convex values;

$\left(\mathrm{H}_{3}\right)$ there exists a positive real number $\mathrm{R}$ such that

$$
R>\frac{\left(2 F_{0} / \Gamma(\alpha)\right)\|g\|_{L^{1}}}{1-(2\|\phi\| / \Gamma(\alpha))\|g\|_{L^{1}}}
$$

where $(2\|\phi\| / \Gamma(\alpha))\|g\|_{L^{1}}<1 / 2, F_{0}=\sup _{t \in[1, e]}|F(t, 0)|$.

Then, the boundary value problem (1) has at least one solution on $[1, e]$.
Proof. Set $X=C([1, e], \mathbb{R})$. Transform the problem (1) into a fixed point problem. Consider the operator $\mathcal{N}: X \rightarrow \mathscr{P}(X)$ defined by

$$
\begin{aligned}
& \mathcal{N} x(t) \\
& =\{h \in C([1, e], \mathbb{R}): h(t)=f(t, x(t)) \\
& \times\left(\frac{1}{\Gamma(\alpha)} \int_{1}^{t}\left(\log \frac{t}{s}\right)^{\alpha-1} \frac{v(s)}{s} d s\right. \\
& \quad-(\log t)^{\alpha-1} \frac{1}{\Gamma(\alpha)} \\
& \left.\times \int_{1}^{e}\left(\log \frac{e}{s}\right)^{\alpha-1} \frac{v(s)}{s} d s\right), \\
& \left.v \in S_{F, x}\right\} .
\end{aligned}
$$

Now we define two operators $\mathscr{A}: X \rightarrow X$ by

$$
\mathscr{A} x(t)=f(t, x(t)), \quad t \in[1, e],
$$

and $\mathscr{B}: X \rightarrow \mathscr{P}(X)$ by

$$
\begin{aligned}
& \mathscr{B} x(t)=\{h \in C([1, e], \mathbb{R}): h(t) \\
&=\frac{1}{\Gamma(\alpha)} \int_{1}^{t}\left(\log \frac{t}{s}\right)^{\alpha-1} \frac{v(s)}{s} d s \\
&-(\log t)^{\alpha-1} \frac{1}{\Gamma(\alpha)} \\
& \times \int_{1}^{e}\left(\log \frac{e}{s}\right)^{\alpha-1} \frac{v(s)}{s} d s, \\
&\left.v \in S_{F, x}\right\} .
\end{aligned}
$$

Observe that $\mathscr{N}(x)=\mathscr{A} x \mathscr{B} x$. We will show that the operators $\mathscr{A}$ and $\mathscr{B}$ satisfy all the conditions of Lemma 7 . For the sake of convenience, we split the proof into several steps.

Step 1. $\mathscr{A}$ is a Lipschitz on $X$; that is, (a) of Lemma 7 holds. Let $x, y \in X$. Then by $\left(\mathrm{H}_{1}\right)$, we have

$$
\begin{aligned}
|\mathscr{A} x(t)-\mathscr{A} y(t)| & =|f(t, x(t))-f(t, y(t))| \\
& \leq \phi(t)|x(t)-y(t)| \\
& \leq\|\phi\|\|x-y\|
\end{aligned}
$$

for all $t \in[1, e]$. Taking the supremum over the interval $[1, e]$, we obtain

$$
\|\mathscr{A} x-\mathscr{A} y\| \leq\|\phi\|\|x-y\|
$$

for all $x, y \in X$. So $\mathscr{A}$ is a Lipschitz on $X$ with Lipschitz constant $\|\phi\|$.

Step 2. The multivalued operator $\mathscr{B}$ is compact and upper semicontinuous on $X$; that is, (b) of Lemma 7 holds. 
First, we show that $\mathscr{B}$ has convex values. Let $u_{1}, u_{2} \in \mathscr{B} x$. Then there are $v_{1}, v_{2} \in S_{F, x}$ such that

$$
\begin{aligned}
u_{i}(t)= & \frac{1}{\Gamma(\alpha)} \int_{1}^{t}\left(\log \frac{t}{s}\right)^{\alpha-1} \frac{v_{i}(s)}{s} d s \\
& -(\log t)^{\alpha-1} \frac{1}{\Gamma(\alpha)} \int_{1}^{e}\left(\log \frac{e}{s}\right)^{\alpha-1} \frac{v_{i}(s)}{s} d s
\end{aligned}
$$

$i=1,2, t \in[1, e]$. For any $\theta \in[0,1]$, we have

$$
\begin{aligned}
\theta u_{1}( & t)+(1-\theta) u_{2}(t) \\
= & \frac{1}{\Gamma(\alpha)} \int_{1}^{t}\left(\log \frac{t}{s}\right)^{\alpha-1} \frac{\left[\theta u_{1}(s)+(1-\theta) u_{2}(s)\right]}{s} d s \\
& -(\log t)^{\alpha-1} \frac{1}{\Gamma(\alpha)} \\
& \times \int_{1}^{e}\left(\log \frac{e}{s}\right)^{\alpha-1} \frac{\left[\theta u_{1}(s)+(1-\theta) u_{2}(s)\right]}{s} d s \\
= & \frac{1}{\Gamma(\alpha)} \int_{1}^{t}\left(\log \frac{t}{s}\right)^{\alpha-1} \frac{\bar{v}(s)}{s} d s \\
& -(\log t)^{\alpha-1} \frac{1}{\Gamma(\alpha)} \int_{1}^{e}\left(\log \frac{e}{s}\right)^{\alpha-1} \frac{\bar{v}(s)}{s} d s
\end{aligned}
$$

where $\bar{v}(t)=\theta v_{1}(t)+(1-\theta) v_{2}(t) \in F(t, x(t))$ for all $t \in[1, e]$. Hence $\theta u_{1}(t)+(1-\theta) u_{2}(t) \in \mathscr{B} x$ and consequently $\mathscr{B} x$ is convex for each $x \in X$. As a result $\mathscr{B}$ defines a multivalued operator $\mathscr{B}: X \rightarrow \mathscr{P}_{\mathrm{cv}}(X)$.

Next we show that $\mathscr{B}$ maps bounded sets into bounded sets in $X$. To see this, let $Q$ be a bounded set in $X$. Then there exists a real number $r>0$ such that $\|x\| \leq r$, for all $x \in Q$.

Now for each $h \in \mathscr{B} x$, there exists a $v \in S_{F, x}$ such that

$$
\begin{aligned}
h(t)= & \frac{1}{\Gamma(\alpha)} \int_{1}^{t}\left(\log \frac{t}{s}\right)^{\alpha-1} \frac{v(s)}{s} d s \\
& -(\log t)^{\alpha-1} \frac{1}{\Gamma(\alpha)} \int_{1}^{e}\left(\log \frac{e}{s}\right)^{\alpha-1} \frac{v(s)}{s} d s .
\end{aligned}
$$

Then, for each $t \in[1, e]$, using $\left(\mathrm{H}_{2}\right)$ we have

$$
\begin{aligned}
|\mathscr{B} x(t)|= & \mid \frac{1}{\Gamma(\alpha)} \int_{1}^{t}\left(\log \frac{t}{s}\right)^{\alpha-1} \frac{v(s)}{s} d s \\
& \quad-(\log t)^{\alpha-1} \frac{1}{\Gamma(\alpha)} \int_{1}^{e}\left(\log \frac{e}{s}\right)^{\alpha-1} \frac{v(s)}{s} d s \mid \\
\leq & \frac{1}{\Gamma(\alpha)} \int_{1}^{t}\left(\log \frac{t}{s}\right)^{\alpha-1} \frac{g(s)}{s} d s \\
& +(\log t)^{\alpha-1} \frac{1}{\Gamma(\alpha)} \int_{1}^{e}\left(\log \frac{e}{s}\right)^{\alpha-1} \frac{g(s)}{s} d s \\
\leq & \frac{2}{\Gamma(\alpha)}\|g\|_{L^{1}} .
\end{aligned}
$$

This further implies that

$$
\|h\| \leq \frac{2}{\Gamma(\alpha)}\|g\|_{L^{1}}
$$

and so $\mathscr{B}(X)$ is uniformly bounded.

Next we show that $\mathscr{B}$ maps bounded sets into equicontinuous sets. Let $Q$ be, as above, a bounded set and $h \in \mathscr{B} x$ for some $x \in Q$. Then there exists a $v \in S_{F, x}$ such that

$$
\begin{aligned}
h(t)= & \frac{1}{\Gamma(\alpha)} \int_{1}^{t}\left(\log \frac{t}{s}\right)^{\alpha-1} \frac{v(s)}{s} d s \\
& -(\log t)^{\alpha-1} \frac{1}{\Gamma(\alpha)} \int_{1}^{e}\left(\log \frac{e}{s}\right)^{\alpha-1} \frac{v(s)}{s} d s, \quad t \in[1, e]
\end{aligned}
$$

Then, for any $\tau_{1}, \tau_{2} \in[1, e]$, we have

$$
\begin{aligned}
\left|h\left(\tau_{2}\right)-h\left(\tau_{1}\right)\right| & \\
\leq & \frac{\|g\|_{L^{1}}}{\Gamma(\alpha)}\left|\int_{1}^{\tau_{1}}\left(\log \frac{\tau_{1}}{s}\right)^{\alpha-1} \frac{1}{s} d s-\int_{1}^{\tau_{2}}\left(\log \frac{\tau_{2}}{s}\right)^{\alpha-1} \frac{1}{s} d s\right| \\
& +\frac{\|g\|_{L^{1}}\left|\left(\log \tau_{2}\right)^{\alpha-1}-\left(\log \tau_{1}\right)^{\alpha-1}\right|}{\Gamma(\alpha)} \int_{1}^{e}\left(\log \frac{e}{s}\right)^{\alpha-1} \frac{1}{s} d s \\
\leq & \frac{\|g\|_{L^{1}}}{\Gamma(\alpha)}\left|\int_{1}^{\tau_{1}}\left[\left(\log \frac{\tau_{1}}{s}\right)^{\alpha-1}-\left(\log \frac{\tau_{2}}{s}\right)^{\alpha-1}\right] \frac{1}{s} d s\right| \\
& +\frac{\|g\|_{L^{1}}}{\Gamma(\alpha)}\left|\int_{\tau_{1}}^{\tau_{2}}\left(\log \frac{\tau_{2}}{s}\right)^{\alpha-1} \frac{1}{s} d s\right| \\
& +\frac{\|g\|_{L^{1}}\left|\left(\log \tau_{2}\right)^{\alpha-1}-\left(\log \tau_{1}\right)^{\alpha-1}\right|}{\Gamma(\alpha)} \int_{1}^{e}\left(\log \frac{e}{s}\right)^{\alpha-1} \frac{1}{s} d s .
\end{aligned}
$$

Obviously the right hand side of the above inequality tends to zero independently of $x \in Q$ as $t_{2}-t_{1} \rightarrow 0$. Therefore, it follows by the Arzelá-Ascoli theorem that $\mathscr{B}$ : $X \rightarrow \mathscr{P}(X)$ is completely continuous.

In our next step, we show that $\mathscr{B}$ has a closed graph. Let $x_{n} \rightarrow x_{*}, h_{n} \in \mathscr{B}\left(x_{n}\right)$ and $h_{n} \rightarrow h_{*}$. Then we need to show that $h_{*} \in \mathscr{B}$. Associated with $h_{n} \in \mathscr{B}\left(x_{n}\right)$, there exists $v_{n} \in S_{F, x_{n}}$ such that, for each $t \in[1, e]$,

$$
\begin{aligned}
h_{n}(t)= & \frac{1}{\Gamma(\alpha)} \int_{1}^{t}\left(\log \frac{t}{s}\right)^{\alpha-1} \frac{v_{n}(s)}{s} d s \\
& -(\log t)^{\alpha-1} \frac{1}{\Gamma(\alpha)} \int_{1}^{e}\left(\log \frac{e}{s}\right)^{\alpha-1} \frac{v_{n}(s)}{s} d s .
\end{aligned}
$$

Thus it suffices to show that there exists $v_{*} \in S_{F, x_{*}}$ such that, for each $t \in[1, e]$,

$$
\begin{aligned}
h_{*}(t)= & \frac{1}{\Gamma(\alpha)} \int_{1}^{t}\left(\log \frac{t}{s}\right)^{\alpha-1} \frac{v_{*}(s)}{s} d s \\
& -(\log t)^{\alpha-1} \frac{1}{\Gamma(\alpha)} \int_{1}^{e}\left(\log \frac{e}{s}\right)^{\alpha-1} \frac{v_{*}(s)}{s} d s .
\end{aligned}
$$


Let us consider the linear operator $\Theta: L^{1}([1, e], \mathbb{R}) \rightarrow$ $C([1, e], \mathbb{R})$ given by

$$
\begin{aligned}
f \longmapsto \Theta(v)(t)= & \frac{1}{\Gamma(\alpha)} \int_{1}^{t}\left(\log \frac{t}{s}\right)^{\alpha-1} \frac{v(s)}{s} d s \\
& -(\log t)^{\alpha-1} \frac{1}{\Gamma(\alpha)} \int_{1}^{e}\left(\log \frac{e}{s}\right)^{\alpha-1} \frac{v(s)}{s} d s .
\end{aligned}
$$

Observe that

$$
\begin{aligned}
\| h_{n}(t) & -h_{*}(t) \| \\
=\| & \frac{1}{\Gamma(\alpha)} \int_{1}^{t}\left(\log \frac{t}{s}\right)^{\alpha-1} \frac{\left(v_{n}(s)-v_{*}(s)\right)}{s} d s \\
& -(\log t)^{\alpha-1} \frac{1}{\Gamma(\alpha)} \\
& \times \int_{1}^{e}\left(\log \frac{e}{s}\right)^{\alpha-1} \frac{\left(v_{n}(s)-v_{*}(s)\right)}{s} d s \| \\
\text { as } n & \longrightarrow \infty,
\end{aligned}
$$

Thus, it follows by Lemma 6 that $\Theta \circ S_{F}$ is a closed graph operator. Further, we have $h_{n}(t) \in \Theta\left(S_{F, x_{n}}\right)$. Since $x_{n} \rightarrow x_{*}$, therefore, we have

$$
\begin{aligned}
h_{*}(t)= & \frac{1}{\Gamma(\alpha)} \int_{1}^{t}\left(\log \frac{t}{s}\right)^{\alpha-1} \frac{v_{*}(s)}{s} d s \\
& -(\log t)^{\alpha-1} \frac{1}{\Gamma(\alpha)} \int_{1}^{e}\left(\log \frac{e}{s}\right)^{\alpha-1} \frac{v_{*}(s)}{s} d s
\end{aligned}
$$

for some $v_{*} \in S_{F, x_{*}}$.

As a result we have that the operator $\mathscr{B}$ is compact and upper semicontinuous operator on $X$.

Step 3. Now we show that $2 M k<1$; that is, (c) of Lemma 7 holds.

This is obvious by $\left(\mathrm{H}_{3}\right)$ since we have $M=\|B(X)\|=$ $\sup \{|\mathscr{B} x|: x \in X\} \leq(2 / \Gamma(\alpha))\|g\|_{L^{1}}$ and $k=\|\phi\|$.

Thus all the conditions of Lemma 7 are satisfied and a direct application of it yields that either conclusion (i) or conclusion (ii) holds. We show that conclusion (ii) is not possible.

Let $u \in \mathscr{E}$ be arbitrary. Then we have, for $\lambda>1, \lambda u \in$ $\mathscr{A} u(t) \mathscr{B} u(t)$. Then there exists $v \in S_{F, x}$ such that, for any $\lambda>$ 1 , one has

$$
\begin{aligned}
u(t)=\lambda^{-1}[f(t, u(t))] & \\
\times & \left(\frac{1}{\Gamma(\alpha)} \int_{1}^{t}\left(\log \frac{t}{s}\right)^{\alpha-1} \frac{v(s)}{s} d s\right. \\
& \left.\quad-(\log t)^{\alpha-1} \frac{1}{\Gamma(\alpha)} \int_{1}^{e}\left(\log \frac{e}{s}\right)^{\alpha-1} \frac{v(s)}{s} d s\right),
\end{aligned}
$$

for all $t \in[1, e]$. Then we have

$$
\begin{aligned}
|u(t)| \leq & \lambda^{-1}|f(t, u(t))| \\
& \times\left(\frac{1}{\Gamma(\alpha)} \int_{1}^{t}\left(\log \frac{t}{s}\right)^{\alpha-1} \frac{|v(s)|}{s} d s\right. \\
& \left.\quad+(\log t)^{\alpha-1} \frac{1}{\Gamma(\alpha)} \int_{1}^{e}\left(\log \frac{e}{s}\right)^{\alpha-1} \frac{|v(s)|}{s} d s\right) \\
\leq & {[|f(t, u(t))-f(t, 0)|+|f(t, 0)|] } \\
& \times\left(\frac{1}{\Gamma(\alpha)} \int_{1}^{t}\left(\log \frac{t}{s}\right)^{\alpha-1} \frac{|v(s)|}{s} d s\right. \\
& \left.\quad+(\log t)^{\alpha-1} \frac{1}{\Gamma(\alpha)} \int_{1}^{e}\left(\log \frac{e}{s}\right)^{\alpha-1} \frac{|v(s)|}{s} d s\right) \\
\leq & {\left[\|\phi\|\|u\|+F_{0}\right] \frac{2}{\Gamma(\alpha)}\|g\|_{L^{1}}, }
\end{aligned}
$$

where we have put $F_{0}=\sup _{t \in[1, e]}|f(t, 0)|$. Then with $\|u\|=R$, we have

$$
R \leq \frac{\left(2 F_{0} / \Gamma(\alpha)\right)\|g\|_{L^{1}}}{1-(2\|\phi\| / \Gamma(\alpha))\|g\|_{L^{1}}} .
$$

Thus condition (ii) of Lemma 7 does not hold by (14). Therefore the operator equation $\mathscr{A} x \mathscr{B} x$ and consequently problem (1) have a solution on $[1, e]$. This completes the proof.

Theorem 10. Assume that $\left(H_{1}\right)$ holds. In addition, one supposes that

(H2) there exists a continuous nondecreasing function $\psi$ : $[0, \infty) \rightarrow(0, \infty)$ and a function $p \in C\left([1, e], \mathbb{R}^{+}\right)$ such that

$$
\begin{aligned}
\|F(t, x)\|_{\mathscr{P}} & :=\sup \{|y|: y \in F(t, x)\} \\
& \leq p(t) \psi(|x|) \text { for each }(t, x) \in[1, e] \times \mathbb{R} ;
\end{aligned}
$$

(H3) there exists a constant $r>0$ such that

$$
r>\frac{\left(2 F_{0} / \Gamma(\alpha)\right)\|p\| \psi(r)}{1-(2\|\phi\| / \Gamma(\alpha))\|p\| \psi(r)},
$$

where

$$
\frac{2\|\phi\|}{\Gamma(\alpha)}\|p\| \psi(r)<\frac{1}{2},
$$

$$
\text { and } F_{0}=\sup _{t \in[1, e]}|F(t, 0)| \text {. }
$$

Then the boundary value problem (1) has at least one solution on $[1, e]$.

Proof. The proof is similar to that of Theorem 9 and is omitted. 
Example 11. Consider the boundary value problem

$$
\begin{gathered}
D^{3 / 2}\left[\frac{x(t)}{(1 / 12) e^{1-t} \tan ^{-1} x+2}\right] \in F(t, x(t)), \quad 1<t<e, \\
x(1)=x(e)=0,
\end{gathered}
$$

where $F:[1, e] \times \mathbb{R} \rightarrow \mathscr{P}(\mathbb{R})$ is a multivalued map given by

$$
t \rightarrow F(t, x)=\left[\frac{|x|^{3}}{10\left(|x|^{3}+3\right)}, \frac{|\sin x|}{9(|\sin x|+1)}+\frac{8}{9}\right]
$$

By condition $\left(\mathrm{H}_{1}\right), \phi(t)=e^{1-t} / 12$ with $\|\phi\|=1 / 12$. For $\tilde{f} \in F$, we have

$$
\begin{gathered}
|\tilde{f}| \leq \max \left(\frac{|x|^{3}}{10\left(|x|^{3}+3\right)}, \frac{|\sin x|}{9(|\sin x|+1)}+\frac{8}{9}\right) \leq 1, \\
x \in \mathbb{R}, \\
\|F(t, x)\|=\sup \{|y|: y \in F(t, x)\} \leq 1=g(t), \quad x \in \mathbb{R} .
\end{gathered}
$$

Clearly,

$$
\frac{2\|\phi\|\|g\|_{L^{1}}}{\Gamma(\alpha)}=\frac{(e-1)}{3 \sqrt{\pi}} \simeq 0.323146<\frac{1}{2}
$$

and $R>24(e-1) /(1+3 \sqrt{\pi}-e)$. Hence all the conditions of Theorem 9 are satisfied and, accordingly, the problem (38) has a solution on $[1, e]$.

\section{Conflict of Interests}

The authors declare that there is no conflict of interests regarding the publication of this paper.

\section{Acknowledgments}

This work was supported by Deanship of Scientific Research (DSR), King Abdulaziz University, Jeddah, Saudi Arabia. The authors thank the referees for their useful comments. Sotiris K. Ntouyas is a member of Nonlinear Analysis and Applied Mathematics (NAAM), Research Group at King Abdulaziz University, Jeddah, Saudi Arabia.

\section{References}

[1] I. Podlubny, Fractional Differential Equations, vol. 198 of Mathematics in Science and Engineering, Academic Press, San Diego, Calif, USA, 1999.

[2] A. A. Kilbas, H. M. Srivastava, and J. J. Trujillo, Theory and Applications of Fractional Differential Equations, vol. 204 of North-Holland Mathematics Studies, Elsevier Science B.V., Amsterdam, The Netherlands, 2006.

[3] J. Sabatier, O. P. Agrawal, and J. A. T. Machado, Eds., Advances in Fractional Calculus: Theoretical Developments and Applications in Physics and Engineering, Springer, Dordrecht, The Netherlands, 2007.
[4] B. Ahmad and J. J. Nieto, "Riemann-Liouville fractional integrodifferential equations with fractional nonlocal integral boundary conditions," Boundary Value Problems, vol. 2011, article 36, 2011.

[5] A. Alsaedi, S. K. Ntouyas, and B. Ahmad, "Existence results for Langevin fractional differential inclusions involving two fractional orders with four-point multiterm fractional integral boundary conditions," Abstract and Applied Analysis, vol. 2013, Article ID 869837, 17 pages, 2013.

[6] D. Băleanu, O. G. Mustafa, and R. P. Agarwal, "On $L^{p}$-solutions for a class of sequential fractional differential equations," Applied Mathematics and Computation, vol. 218, no. 5, pp. 20742081, 2011.

[7] R. P. Agarwal and B. Ahmad, "Existence theory for anti-periodic boundary value problems of fractional differential equations and inclusions," Computers \& Mathematics with Applications, vol. 62, no. 3, pp. 1200-1214, 2011.

[8] J. R. Graef, L. Kong, and Q. Kong, "Application of the mixed monotone operator method to fractional boundary value problems," Fractional Differential Calculus, vol. 2, no. 1, pp. 87-98, 2012.

[9] F. T. Akyildiz, H. Bellout, K. Vajravelu, and R. A. Van Gorder, "Existence results for third order nonlinear boundary value problems arising in nano boundary layer fluid flows over stretching surfaces," Nonlinear Analysis: Real World Applications, vol. 12, no. 6, pp. 2919-2930, 2011.

[10] B. Ahmad and J. J. Nieto, "Sequential fractional differential equations with three-point boundary conditions," Computers \& Mathematics with Applications, vol. 64, no. 10, pp. 3046-3052, 2012.

[11] Z. Bai and W. Sun, "Existence and multiplicity of positive solutions for singular fractional boundary value problems," Computers \& Mathematics with Applications, vol. 63, no. 9, pp. 1369-1381, 2012.

[12] R. Sakthivel, N. I. Mahmudov, and J. J. Nieto, "Controllability for a class of fractional-order neutral evolution control systems," Applied Mathematics and Computation, vol. 218, no. 20, pp. 10334-10340, 2012.

[13] B. Ahmad and J. J. Nieto, "Anti-periodic fractional boundary value problems with nonlinear term depending on lower order derivative," Fractional Calculus and Applied Analysis, vol. 15, no. 3, pp. 451-462, 2012.

[14] R. P. Agarwal, D. O’Regan, and S. Staněk, "Positive solutions for mixed problems of singular fractional differential equations," Mathematische Nachrichten, vol. 285, no. 1, pp. 27-41, 2012.

[15] J. Wang, Y. Zhou, and M. Medveud, "Qualitative analysis for nonlinear fractional differential equations via topological degree method," Topological Methods in Nonlinear Analysis, vol. 40, no. 2, pp. 245-271, 2012.

[16] A. Cabada and G. Wang, "Positive solutions of nonlinear fractional differential equations with integral boundary value conditions," Journal of Mathematical Analysis and Applications, vol. 389, no. 1, pp. 403-411, 2012.

[17] L. Zhang, B. Ahmad, G. Wang, and R. P. Agarwal, "Nonlinear fractional integro-differential equations on unbounded domains in a Banach space," Journal of Computational and Applied Mathematics, vol. 249, pp. 51-56, 2013.

[18] W.-X. Zhou, Y.-D. Chu, and D. Băleanu, "Uniqueness and existence of positive solutions for a multi-point boundary value problem of singular fractional differential equations," Advances in Difference Equations, vol. 2013, article 114, 2013. 
[19] B. Ahmad, S. K. Ntouyas, and A. Alsaedi, "A study of nonlinear fractional differential equations of arbitrary order with Riemann-Liouville type multistrip boundary conditions," Mathematical Problems in Engineering, vol. 2013, Article ID 320415, 9 pages, 2013.

[20] B. Ahmad and S. K. Ntouyas, "Existence results for higher order fractional differential inclusions with multi-strip fractional integral boundary conditions," Electronic Journal of Qualitative Theory of Differential Equations, no. 20, 19 pages, 2013.

[21] M. Faieghi, S. Kuntanapreeda, H. Delavari, and D. Baleanu, "LMI-based stabilization of a class of fractional-order chaotic systems," Nonlinear Dynamics, vol. 72, no. 1-2, pp. 301-309, 2013.

[22] J. J. Nieto, A. Ouahab, and P. Prakash, "Extremal solutions and relaxation problems for fractional differential inclusions," Abstract and Applied Analysis, vol. 2013, Article ID 292643, 9 pages, 2013.

[23] D. O’Regan and S. Staněk, "Fractional boundary value problems with singularities in space variables," Nonlinear Dynamics, vol. 71, no. 4, pp. 641-652, 2013.

[24] J. Wang, Y. Zhou, and W. Wei, "Fractional sewage treatment models with impulses at variable times," Applicable Analysis: An International Journal, vol. 92, no. 9, pp. 1959-1979, 2013.

[25] C. Zhai and M. Hao, "Mixed monotone operator methods for the existence and uniqueness of positive solutions to RiemannLiouville fractional differential equation boundary value problems," Boundary Value Problems, vol. 2013, article 85, 2013.

[26] B. Ahmad and S. K. Ntouyas, "Existence results for fractional differential inclusions arising from real estate asset securitization and HIV models," Advances in Difference Equations, vol. 2013, article 216, 2013.

[27] B. Ahmad and S. K. Ntouyas, "New existence results for differential inclusions involving Langevin equation with two indices," Journal of Nonlinear and Convex Analysis, vol. 14, no. 3, pp. 437-450, 2013.

[28] J. Wang, Y. Zhou, and M. Fečkan, "On the nonlocal Cauchy problem for semilinear fractional order evolution equations," Central European Journal of Mathematics, vol. 12, no. 6, pp. 911922, 2014.

[29] J. R. Graef, L. Kong, and M. Wang, "Existence and uniqueness of solutions for a fractional boundary value problem on a graph," Fractional Calculus and Applied Analysis, vol. 17, no. 2, pp. 499510, 2014.

[30] F. Punzo and G. Terrone, "On the Cauchy problem for a general fractional porous medium equation with variable density," Nonlinear Analysis: Theory, Methods \& Applications, vol. 98, pp. 27-47, 2014.

[31] X. Liu, Z. Liu, and X. Fu, "Relaxation in nonconvex optimal control problems described by fractional differential equations," Journal of Mathematical Analysis and Applications, vol. 409, no. 1, pp. 446-458, 2014.

[32] K. Razminia, A. Razminia, and J. A. Tenreiro Machado, "Analysis of diffusion process in fractured reservoirs using fractional derivative approach," Communications in Nonlinear Science and Numerical Simulation, vol. 19, no. 9, pp. 3161-3170, 2014.

[33] S. Choudhary and V. Daftardar-Gejji, "Nonlinear multi-order fractional differential equations with periodic/anti-periodic boundary conditions," Fractional Calculus and Applied Analysis, vol. 17, no. 2, pp. 333-347, 2014.

[34] J. Hadamard, "Essai sur l'etude des fonctions donnees par leur developpment de Taylor," Journal de Mathématiques Pures et Appliquées Série, vol. 8, pp. 101-186, 1892.
[35] B. C. Dhage, "Existence results for neutral functional differential inclusions in Banach algebras," Nonlinear Analysis: Theory, Methods \& Applications, vol. 64, no. 6, pp. 1290-1306, 2006.

[36] M. M. El Borai and M. I. Abbas, "On some integro-differential equations of fractional orders involving Carathéodory nonlinearities," International Journal of Modern Mathematics, vol. 2, no. 1, pp. 41-52, 2007.

[37] Y. Zhao, S. Sun, Z. Han, and Q. Li, "Theory of fractional hybrid differential equations," Computers \& Mathematics with Applications, vol. 62, no. 3, pp. 1312-1324, 2011.

[38] S. Sun, Y. Zhao, Z. Han, and Y. Li, "The existence of solutions for boundary value problem of fractional hybrid differential equations," Communications in Nonlinear Science and Numerical Simulation, vol. 17, no. 12, pp. 4961-4967, 2012.

[39] M. R. Sidi Ammi, E. H. El Kinani, and D. F. M. Torres, "Existence and uniqueness of solutions to functional integro-differential fractional equations," Electronic Journal of Differential Equations, no. 103, 9 pages, 2012.

[40] B. C. Dhage and S. K. Ntouyas, "Existence results for boundary value problems for fractional hybrid differentialinclucions," Topological Methods in Nonlinear Analysis. In press.

[41] M. D. Kassim and N.-E. Tatar, "Well-posedness and stability for a differential problem with Hilfer-Hadamard fractional derivative," Abstract and Applied Analysis, vol. 2013, Article ID 605029, 12 pages, 2013.

[42] B. Ahmad, S. K. Ntouyas, and A. Alsaedi, "On fractional differential inclusions with anti-periodic type integral boundary conditions," Boundary Value Problems, vol. 2013, 82 pages, 2013.

[43] B. Ahmad and S. K. Ntouyas, "A fully Hadamard type integral boundary value problem of a coupled system of fractional differential equations," Fractional Calculus and Applied Analysis, vol. 17, no. 2, pp. 348-360, 2014.

[44] B. Ahmad and S. K. Ntouyas, "On Hadamard fractional integrodifferential boundary value problems," Journal of Applied Mathematics and Computing, 2014.

[45] K. Deimling, Multivalued Differential Equations, vol. 1 of de Gruyter Series in Nonlinear Analysis and Applications, Walter de Gruyter, Berlin, Germany, 1992.

[46] S. Hu and N. S. Papageorgiou, Handbook of Multivalued Analysis. Vol. I, Theory, vol. 419 of Mathematics and its Applications, Kluwer Academic, Dordrecht, The Netherlands, 1997.

[47] A. Lasota and Z. Opial, "An application of the Kakutani-Ky Fan theorem in the theory of ordinary differential equations," Bulletin de l'Académie Polonaise des Sciences: Série des Sciences Mathématiques, Astronomiques et Physiques, vol. 13, pp. 781-786, 1965. 


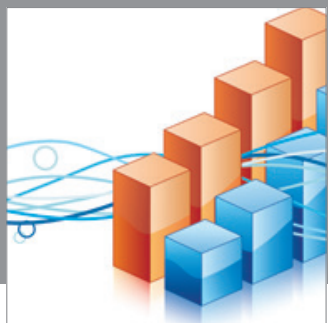

Advances in

Operations Research

mansans

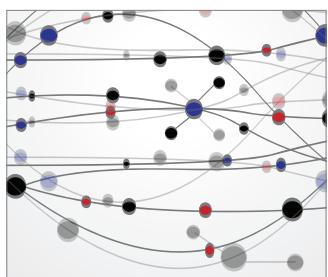

The Scientific World Journal
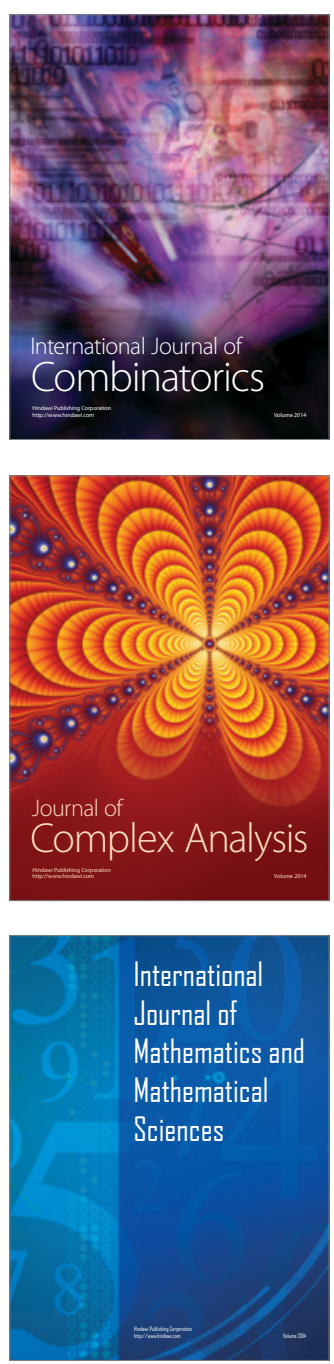
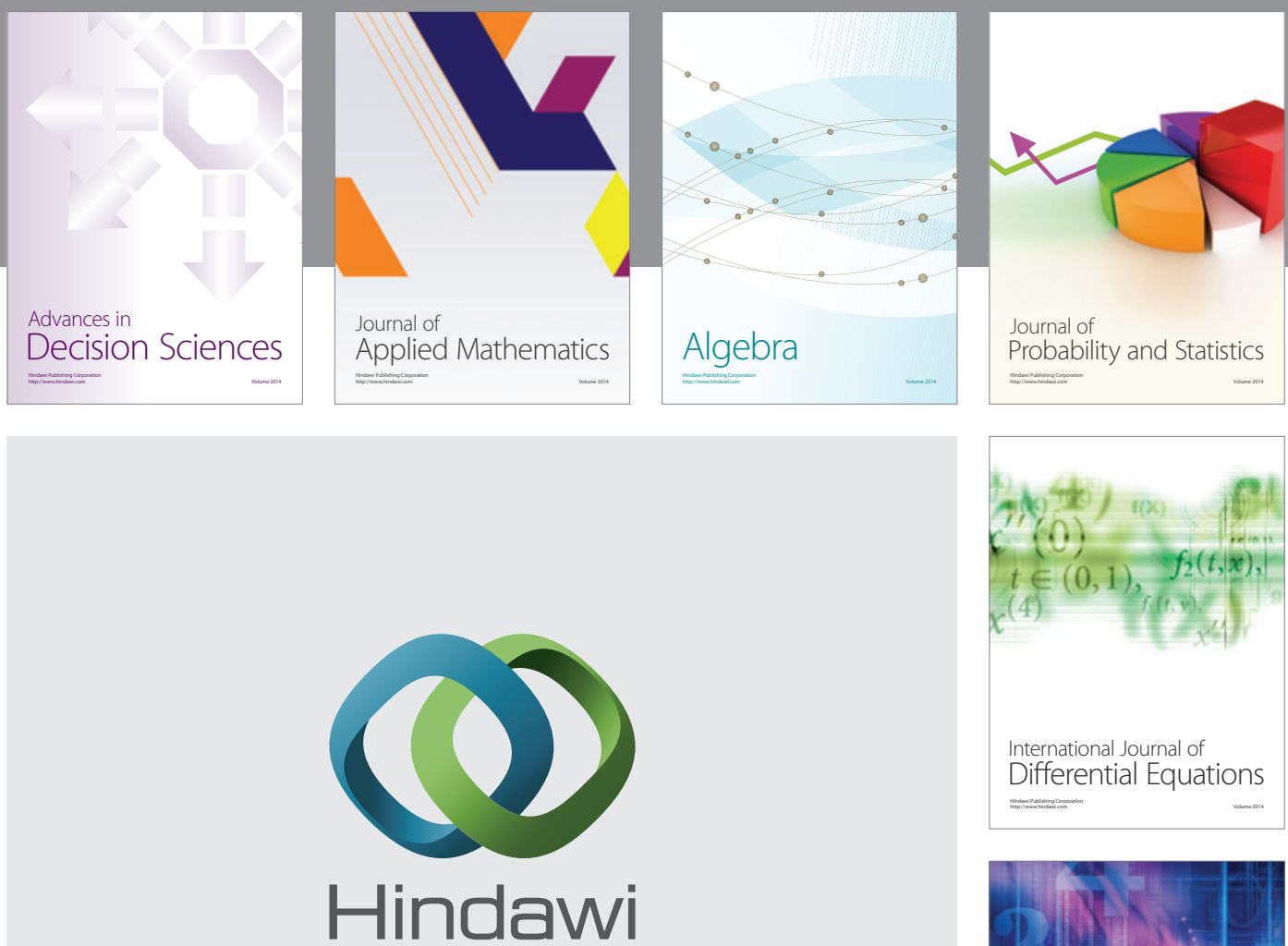

Submit your manuscripts at http://www.hindawi.com
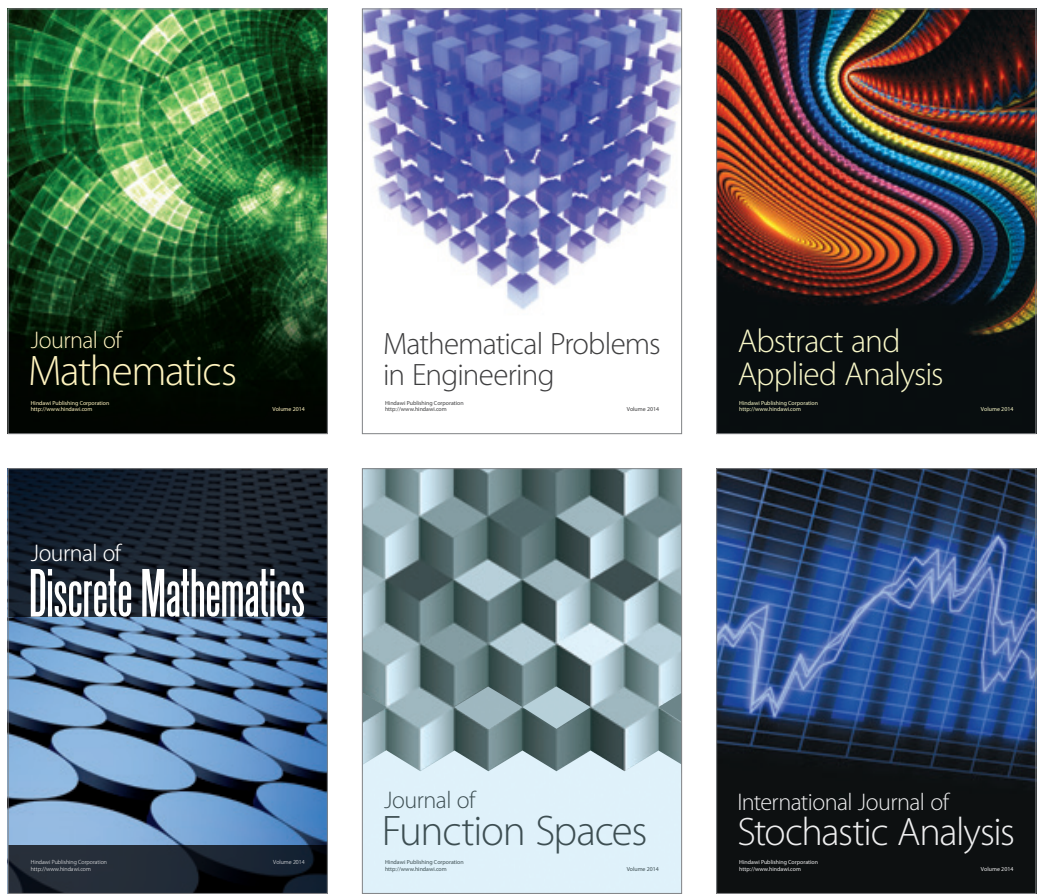

Journal of

Function Spaces

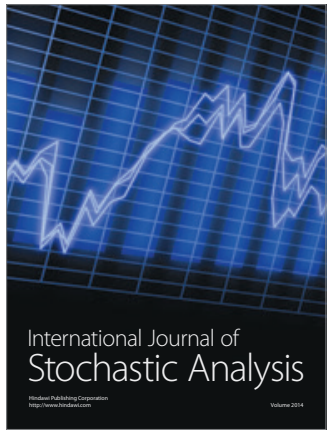

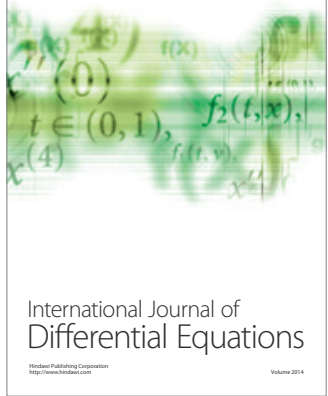
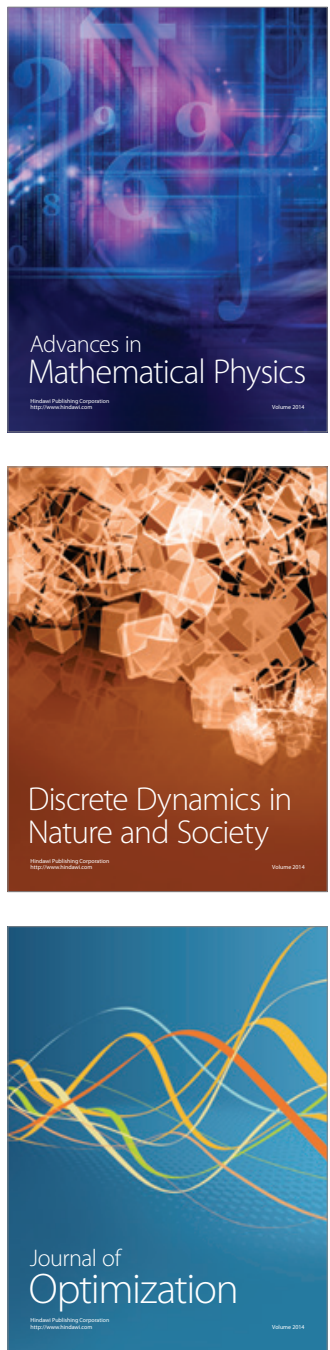